\title{
Communauté d'apprentissage et communauté de pratique en ligne : le processus réflexif dans la formation des formateurs
}

\section{Thierry Chanier}

Université de Franche-Comté, FRANCE

thierry.chanier@univ-fcomte.fr

\section{Jacques Cartier}

Division de la Formation, Rectorat de Besançon, FRANCE

jacques.cartier@ac-besancon.fr

Réflexion pédagogique

\section{Résumé}

Saisissant l'occasion offerte par de récentes politiques éducatives qui orientent la formation des enseignants vers le travail collectif en réseaux, nous abordons ici la délicate question de l'association entre pratique réflexive et participation à des communautés de pratique en ligne. Motivés par le développement professionnel de l'enseignant, nous montrons l'intérêt de concevoir des formations qui ont pour enjeux d'être un terreau favorisant l'émergence de communautés de pratique en ligne et d'ouvrir les praticiens à de nouvelles postures pédagogiques. Nous présentons un paradigme qui articule quatre notions : réflexion sur les pratiques, cadre dialogique pour le processus réflexif, communauté d'apprentissage et communauté de pratique. Nous décrivons un dispositif de travail réflexif intégré au sein d'une formation collaborative avec ses supports d'expression, son scénario et son processus dialogique. L'ensemble est illustré par une étude de cas portant sur une formation de formateurs qui s'est répétée sur deux années consécutives.

\section{Summary}

Recent educational policies are oriented towards the training of teachers for online collaborative work. We size this opportunity to examine the delicate relationship between reflective practice and community of practice. Keeping in mind the professional development of teachers, we stress the interest of developing trainings which support the emergence of communities of practice and raise the awareness about new instructional strategies. We introduce a paradigm which articulates four notions, namely reflection on practice, dialogue in the reflective process, learning community and community of practice. We describe a framework for reflection integrated into a collaborative learning process, its specific media of discourse, scenario and dialogical process. A case study illustrates our approach.

(C)Auteur(s). Cette œuvre, disponible à http://ritpu.ca/IMG/pdf/cartier.pdf, est mise à disposition selon les termes de la licence Creative Commons Attribution - Pas de Modification 2.5 Canada :

http://creativecommons.org/licences/by-nd/2.5/ca/deed.fr 


\section{Pratique réflexive, communauté de pratique et développement professionnel}

Depuis les travaux de Schön à la fin des années 1980, la pratique réflexive est perçue comme une des voies du développement professionnel des enseignants, qu'ils soient élèves enseignants (preservice teacher) ou déjà praticiens (in-service teacher) (Ferraro, 2000). Issue d'un passé plus récent (fin des années 1990), la constitution de communautés de praticiens en ligne est présentée, en Europe comme sur le continent américain, dans une même perspective (Barab, Kling et Gray, 2004; Daele et Charlier, 2002). L'article est subdivisé en deux parties: la première fait état de la littérature scientifique francophone et anglophone dans les domaines de l'intégration et de la formation aux TIC dans la pratique enseignante; la seconde présente notre recherche proprement dite, exposant successivement la méthodologie, les principaux résultats ainsi que leur analyse.

\subsection{Les communautés de pratique enseignantes}

Par «communauté de pratique» (CdP), nous comprenons, comme Barab, Makinster et Scheckler (2004, p. 55), qui s'inscrivent dans l'approche de Lave et Wenger (1991), « un réseau social persistant et actif d'individus qui partagent et développent un fonds de connaissances, un ensemble de croyances, de valeurs, une histoire et des expériences concentrées sur une pratique commune et/ou une entreprise commune ». L'Internet, les réseaux informatiques et les collecticiels associés semblent offrir l'environnement de travail approprié au développement de ces réseaux sociaux, un environnement où $l^{\prime}$ on peut tout à la fois échanger des ressources éducatives et discuter les approches éducatives, et où s'actualise l'identité professionnelle de l'enseignant. Réfléchir sur son enseignement et partager avec les autres en vue d'améliorer son vécu et ses pratiques pédagogiques fondent l'argumentaire des partisans de la pratique réflexive, comme de ceux des $\mathrm{CdP}$.

Dans l'approche $\mathrm{CdP}$, une relation explicite est établie entre l'enseignant et son rapport à la communauté, d'une part, et ses pratiques pédagogiques en classe, d'autre part.

"Teachers who played important roles in larger educational community were more likely to use constructivist and collaborative instructional strategies in their classrooms, while teachers who became less involved in collaborative activities with other colleagues were more likely to use direct instruction and individualized learning tasks » (Schlager et Fusco, 2004).

Une pratique qui ne se donne pas à voir, ni à être discutée (private practice), couplée à une vision de l'enseignant isolé dans sa classe (isolated in their classrooms), est mise à l'index des deux côtés de l'Atlantique. La formation des enseignants aux technologies de l'information et de la communication pour l'éducation (TICE) spécialisées sur les réseaux devient donc une occasion de travailler en profondeur sur la rénovation du métier d'enseignant. Aux États-Unis, par exemple, une politique menée au niveau des États cherche à développer ces $\mathrm{CdP}$ en ligne (Schlager et Fusco, 2004). En France, les auteurs du référentiel C2Ienseignant (Ministère de l'éducation nationale, de l'enseignement supérieur et de la recherche [MENESR], 2005) recherchent le même but avec le soutien du ministère de l'Éducation. L'ensemble de compétences décrit dans ce référentiel concerne tous les enseignants, qu'ils soient élèves enseignants ou en poste. Dépassant le simple niveau de littératie informatique des référentiels précédents (destinés aux collégiens, lycéens, étudiants, etc. - pour une vue synoptique de ces référentiels TICE, voir Reffay et Chanier, 2005), le C2I-enseignant invite le praticien à rentrer dans une démarche associant étroitement son intervention en classe à son intervention dans sa communauté professionnelle, 
en accordant de l'importance à la dimension sociale de l'apprentissage, le tout dans un environnement de travail s'appuyant largement sur les réseaux informatiques et les collecticiels.

\subsection{Un rapprochement non systéma- tique entre pratique reflexive et $\mathrm{CdP}$}

Les objectifs partiellement communs relevés chez les tenants de la pratique réflexive, d'une part, et chez ceux des $\mathrm{CdP}$, d'autre part, ne conduisent pas pour autant à un rapprochement systématique des deux notions. Le terme réflexif (et ses dérivés) est ainsi absent de la table des matières de l'ouvrage de Barab, Kling et al. (2004). Perrenoud (2001), l'un des porte-parole en France de la pratique réflexive dans le métier d'enseignant, la positionne comme une démarche avant tout individuelle. Le qualificatif « individuel» ne porte pas seulement sur la démarche (ce qui est indéniable), mais également sur le cadre de travail de la mise en pratique réflexive. L'analyse collective des pratiques, discutée dans son livre, est introduite davantage comme une façon parmi d'autres de s'initier à cette pratique réflexive. Aucun lien avec un mouvement d'intégration dans une $\mathrm{CdP}$, au sens évoqué ici, n'est envisagé. L'auteur écarte même l'idée d'associer développement d'une démarche réflexive et développement $d$ 'une nouvelle démarche pédagogique inspirée des recherches en didactique (Perrenoud, 2001, p. 196).

Les points de tension entre individu et groupe sont potentiellement nombreux. Du côté de la pratique réflexive, il est essentiel de créer un environnement de confiance où la prise de risque individuelle peut avoir lieu. Le faire en associant l'individu à des groupes où transparaissent les relations disciplinaires, hiérarchiques et de proximité quotidienne de travail contrecarrerait cet engagement. Du côté des CdP en ligne, l'ouvrage de Daele et Charlier (2002) laisse voir certains exemples de communautés où s'élaborent des normes professionnelles contraignantes et où apparaissent des enjeux de pouvoir en rapport avec l'institution éducative. Pour les auteurs du collectif coordonné par Barab, Kling et al. (2004), la recherche dans les $\mathrm{CdP}$ en est encore à ses débuts. Ils rappellent le coût d'entrée dans une communauté, non négligeable pour un individu (sur les plans de l'habitus, de la technologie, etc.) et la non-automaticité de l'évolution d'un groupe de praticiens en CdP. Ils s'interrogent sur les difficultés à faire participer un large nombre d'enseignants à une communauté, sur les outils permettant de mesurer si une collectivité en ligne est ou non une $\mathrm{CdP}$ et sur son impact dans les pratiques en classe. Notons qu'il semble nettement plus facile de développer une $\mathrm{CdP}$ en ligne à partir d'une communauté existant déjà en présentiel (Barab, Kling et al., 2004, p.9). Les recherches des auteurs s'orientent vers la conception (design) d'environnements technologiques dotés de fonctions optimisées pour le travail de ces groupes, ainsi que de tâches qui renforcent expériences et pratiques.

\subsection{Une approche plus récente mentionnant pratique réflexive et $\mathrm{CdP}$}

Dans une version plus récente de leurs travaux, Daele et Charlier (2006) associent réflexivité, communauté et développement professionnel.

«Plusieurs auteurs considèrent de plus en plus le développement professionnel des enseignants comme un processus alimenté non seulement par la formation initiale et la formation continue, mais également par les pairs et par la réflexivité personnelle dans d'autres situations, professionnelles ou privées » (Daele et Charlier, 2006, p. 90).

Dans cette citation est évoqué le contexte social dans lequel se déroule la réflexivité ainsi que son lien avec le développement professionnel. On notera l'acception particulière du terme «formation continue » qui ici, comme dans le reste de l'article, semble référer aux formations de type traditionnel. 
Plus loin, les auteurs développent un modèle de développement professionnel d'un enseignant au sein d'une communauté virtuelle (Daele et Charlier, 2006, p. 98). Le schéma cadre assez bien en fait avec les modèles de communautés discutés dans l'ouvrage de Barab, Kling et al. (2004). La réflexion y est bien mentionnée d'un côté, les échanges de l'autre, mais l'ensemble ne fait pas l'objet d'un développement particulier. Enfin, l'action est essentiellement tournée vers les pratiques de classe et accorde donc une priorité aux enjeux disciplinaires.

\subsection{Une première étude de cas notable}

Portons maintenant notre regard sur l'une des premières études reliant explicitement pratique réflexive et CdP. Makinster, Barab, Harwood et Andersen (2006) se sont intéressés à la nature de la pratique réflexive en ligne d'un public d'élèves enseignants en science et à leurs ressentis sur ce travail métacognitif, en plongeant ces sujets dans trois modalités d'interaction sociale différentes. Pour tous, le travail de réflexion se construit autour d'un journal qui doit répondre à un questionnement préétabli, sous forme d'une succession de messages postés dans un forum en ligne. La réflexion s'élabore au cours de leurs premiers stages en position d'enseignant responsable d'une classe. Dans la première modalité, chaque élève enseignant du groupe correspondant écrit dans un forum privé en interaction avec son enseignant tuteur de stage. Dans la seconde modalité, une autre partie du public écrit son journal en dialoguant dans un forum ouvert aux pairs. Dans la troisième, le reste $\mathrm{du}$ public intervient dans un forum ouvert comme précédemment aux pairs, mais en plus aux membres de la CdP des enseignants de science de l'État d'Indiana aux États-Unis. Tous ces forums sont ouverts en utilisant un des outils de communication de la plate-forme ILF développée pour cette $\mathrm{CdP}$. De cette étude (menée à petite échelle), il ressort qu'à qualité de contenu réflexif relativement équivalent dans les trois modalités, seuls les élèves enseignants ayant développé leur réflexion suivant la troisième modalité avaient réellement perçu l'intérêt d'une telle démarche. Ils avaient l'impression de pouvoir être lus et de pouvoir échanger avec un public d'enseignants ayant plus d'expérience qu'eux-mêmes. Les élèves du premier groupe (échange privé) avaient tous une perception très négative de la tâche, ce qui constitue un échec de cette formation à l'entrée dans une pratique réflexive censée se poursuivre au long de la carrière d'enseignant.

Cette étude suggère que, même si la pensée réflexive peut s'articuler étroitement avec l'acte d'écriture, la nature de l'écriture (et sa valorisation) change radicalement si son produit est destiné à être lu par d'autres, qui éventuellement peuvent $\mathrm{y}$ répondre ${ }^{1}$. Il semble intéressant de dépasser le cadre esquissé par Schön pour inscrire la pratique réflexive sur l'enseignement et l'apprentissage dans un cadre dialogique ayant un lien social avec la CdP de l'individu. La question de la taille du cadre (c.-àd. du nombre et des modalités de participation des autres intervenants) reste bien sûr critique, le développement d'une pensée réflexive étant au départ une démarche de l'individu qui peut rapidement se sentir peu enclin à développer son point de vue personnel devant un large auditoire. Notons que la question de la nature des outils technologiques (ici le forum) et de leur scénarisation est à nouveau posée.

\subsection{Notre démarche}

La mission qui incombe en France aux DIFOR ${ }^{2}$ est de contribuer à former des enseignants en poste au secondaire (collège et lycée) sur une partie du référentiel C2I, en particulier sur les points «travail en réseau avec l'utilisation des outils de travail collaboratif; conception et préparation de contenus d'enseignement et de situations d'apprentissage; mise en œuvre pédagogique en présentiel et à distance ». À l'académie de Besançon, nous avons décidé de concevoir une formation, dénommée Inti, 
entièrement à distance, à la fois pour des raisons de choix pédagogiques (mettre les individus en vraie situation de FAD) et par nécessité (dispersion des enseignants dans l'académie, ouverture de la formation académique sur le plan national, formation se déroulant sans mise en disponibilité particulière). Cette formation vise, d'autre part, à avoir un effet démultiplicateur au sens où des participants aux premières sessions doivent sortir les futurs tuteurs en ligne d'autres formations. Inti contribue à former des tuteurs en ligne en agissant sur deux plans distincts : les enseignants, placés en situation d'apprenants dans un groupe, interagissent avec un tuteur dont ils doivent analyser le rôle; une partie des tuteurs sont d'anciens apprenants qui prennent leur première responsabilité de groupe. Les enjeux de ce type de formation convergent vers ceux qui ont été évoqués dans les sections précédentes.

Nous avons donc choisi de concevoir une formation de type collaboratif au cours de laquelle les enseignants-apprenants répartis en groupes de taille réduite, animés par un tuteur, devaient construire un projet d'utilisation des TICE dans un contexte pédagogique, suivant un modèle inspiré de LearnNett. Chaque groupe définit son projet, en respectant un même cahier des charges (Cartier et Chanier, 2006). L'occasion nous en étant offerte, nous avons mutualisé cette formation avec un module d'un diplôme universitaire, le master FOAD (formation ouverte et à distance, niveau bac $+5)$. Le public «étudiant» du master est pour moitié composé d'élèves formateurs et pour moitié de formateurs en reconversion professionnelle. Au terme «étudiant», nous préférerons celui de «stagiaire ». Il représente mieux ce public mêlant personnes en formations initiale et continue. Tous doivent devenir ingénieurs pédagogiques, concepteurs, animateurs de formation alliant présentiel et distance. L'intérêt de mélanger ainsi ces deux publics de formateurs (que nous dénommerons «apprenants» lorsque nous y référerons durant les sessions Inti, sans chercher à distinguer si les personnes viennent de la DIFOR ou du master) est d'écarter d'emblée la prégnance des enjeux disciplinaires, ou des rapports hiérarchiques professionnels, d'apporter un mélange de cultures d'apprentissage et institutionnelles qui évite la référence à un modèle unique stéréotypique d'enseignement, et, enfin, de mélanger les niveaux de compétences en dispositifs technologiques FOAD. Ferraro (2000) rappelle l'intérêt de ce mélange des cultures pour le développement professionnel des enseignants en faisant référence au programme d'un institut de formation décrit par Wilhelm et al. (1996) :

«[...] a professional development institute that offers teacher interns an opportunity to explore attitudes [...] and reflect on the ethical implication of practice in classrooms with cultural compositions vastly different from their previous experiences. By its nature, this kind of professional development institute causes teachers to step back and critically reflect not only on how they teach, but also on why they teach in a particular way. "

En contrepartie, cette hétérogénéité introduit des risques indéniables de distorsion dans les groupes, notamment quant aux différences de disponibilités. Les étudiants du master sont dans un cursus d'études supérieures. Ils consacrent leur année à cette formation. Les apprenants DIFOR sont volontaires. Ils prennent sur leur temps personnel pour participer à Inti.

Les recherches sur l'apprentissage collaboratif en ligne ont nettement montré que la transformation d'un groupe en véritable communauté d'apprentissage, avec la force du lien social et de l'intentionnalité de rassemblement que cela implique, n'est pas automatique (Henri et Pudelko, 2002) : risque d'abandons individuels, d'anomie de groupe à la suite de conflits internes ou d'un manque de cohésion, etc. C'est la raison pour laquelle nous faisons l'hypothèse que plonger ces enseignants/formateurs dans la position 
d'apprenants, avec l'objectif de se constituer en communauté d'apprentissage autour d'un projet collaboratif, va représenter une expérience et mobiliser des connaissances et un ensemble de valeurs susceptibles de leur ouvrir la porte d'entrée à des $\mathrm{CdP}$, communautés dans lesquelles la force des liens évoquée précédemment est encore plus forte que dans celle de l'apprentissage (Henri et Pudelko, 2002). Cette approche s'appuie sur une démarche initialement mise en place pour des formations d'enseignants d'université (Reffay et Chanier, 2005). Nous espérons ainsi répondre aux obstacles notés par les recherches étatsuniennes évoquées précédemment quant à la difficulté de faire entrer des enseignants dans une CdP en ligne lorsque cette communauté n'existait pas auparavant.

Par ailleurs, Henri et Lundgren-Cayrol ont bien analysé les relations entre apprentissage individuel et apprentissage en groupe, qui sont au cœur de la démarche collaborative en ligne (2001, p. 42). Tirer l'expérience d'un vécu collaboratif n'implique donc pas seulement pour un apprenant d'avoir produit collectivement, mais aussi d'avoir perçu et compris la progression de son rôle personnel dans la communauté d'apprentissage. Pour ce faire, une mise en posture réflexive s'impose. Dans la première version de la formation Inti en 2004-2005, nous avions invité les participants à tenir un journal de bord individuel et à nous remettre un rapport réflexif après le rendu des travaux collectifs. Cette étape de la formation est passée relativement inaperçue et a connu une participation peu importante des enseignants de la DIFOR. Le choix des outils (privés) et l'absence de construction dialogique nous avaient placés dans une situation comparable à celle qui est évoquée par Makinster et al. (2006) dans sa première modalité expérimentale.

Lors de la formation Inti 2005-2006, il nous est apparu nécessaire de revoir complètement cette

Figure 1. Vue d'ensemble du processus réflexif dans la formation Inti

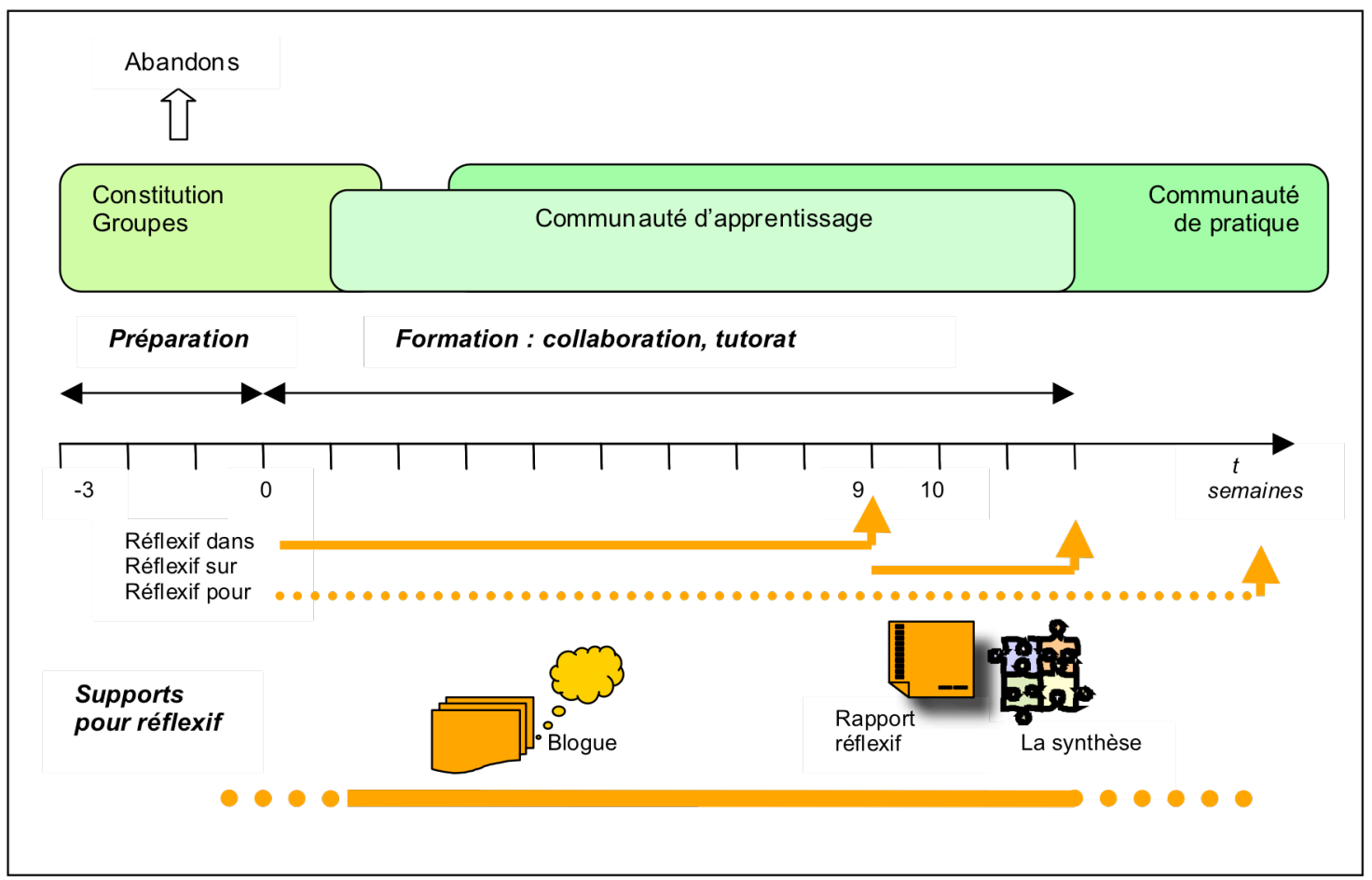


partie réflexive en la dotant d'outils et d'une scénarisation spécifique avec un double enjeu: d'une part, compléter la formation collaborative avec un travail de réflexion individuelle pour améliorer son efficacité; d'autre part, introduire ces enseignants à la pratique réflexive vue comme moyen de développement tout au long de leur carrière professionnelle, pratique devant s'inscrire dans une vie au sein de CdP en ligne.

C'est cette scénarisation spécifique de la mise en place d'une pratique réflexive dans une formation de formateurs que nous décrivons ici à travers l'étude du cas Inti 2005-2006. durant laquelle chaque participant prend connaissance du guide pédagogique de la formation (planning, critères de qualité requis pour le projet, description du rôle des apprenants et tuteurs, etc.) et accomplit la préparation technique pour accéder aux trois espaces technologiques de l'environnement Inti, à savoir : un blogue individuel, une plate-forme asynchrone de téléformation (en l'occurrence Dokeos $\AA$, avec forums, agenda, dépôt de documents, etc.) et une plate-forme synchrone (Centra ${ }^{\circledR}$ avec ses salles « virtuelles » équipées d'audio, vidéo, tableau blanc, etc.). Les groupes vont également être constitués sur la base d'informations transmises pendant cette même période par les participants (notamment les

Figure 2. Processus dialogique dans le réflexif s'offrant à chaque apprenant et à chaque tuteur. Le sens des flèches correspond aux droits d'écriture/lecture.

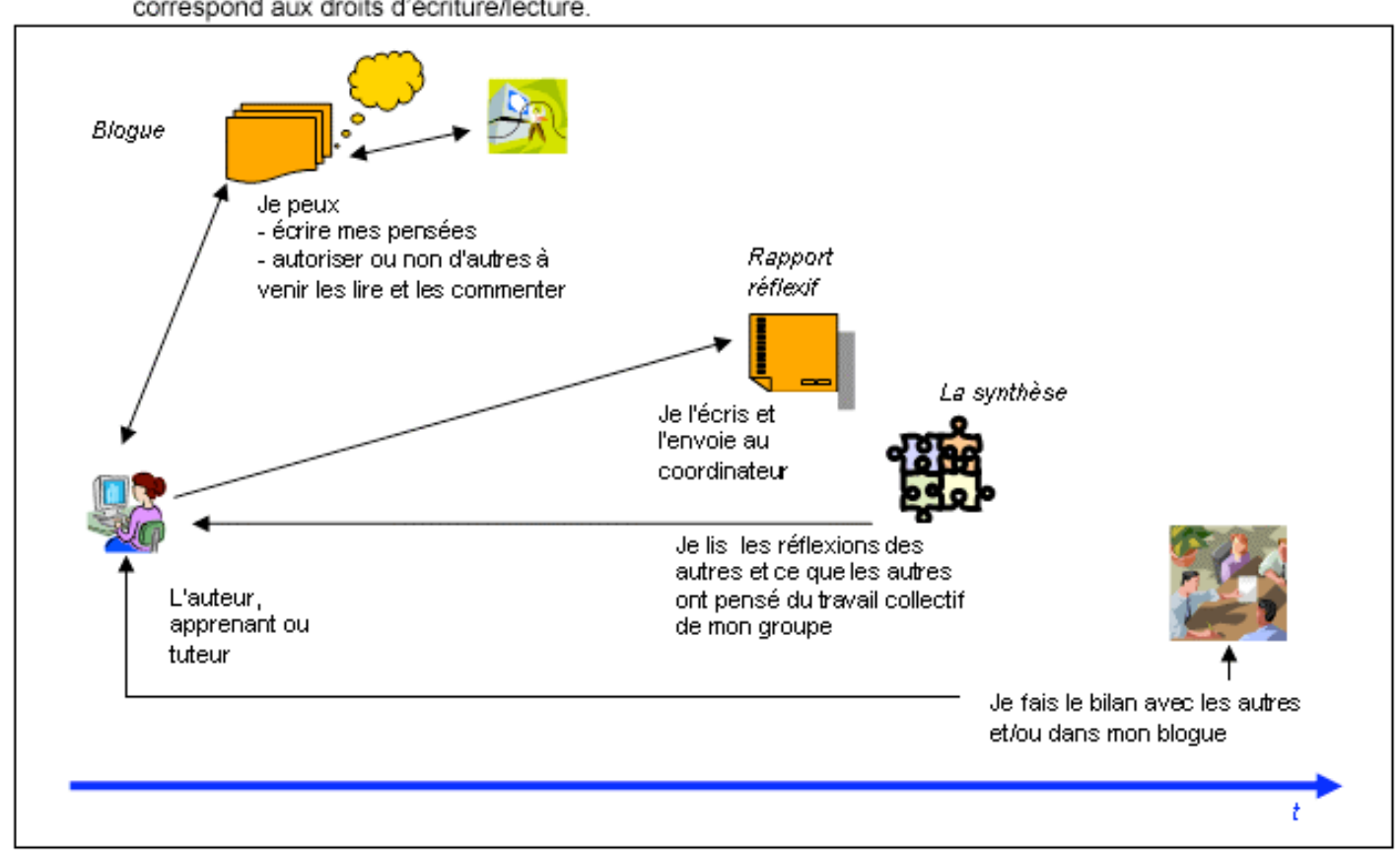

\section{La conception du dispositif réflexif au sein de la formation Inti}

La Figure 1 apporte une vue d'ensemble sur la façon dont le processus réflexif a été scénarisé tout au long de la session de formation Inti.

\subsection{Le travail collaboratif}

Avant le début de la session proprement dite se déroule une étape de préparation (semaines s-3 à s0) disponibilités pour les réunions synchrones). Un tuteur, provenant soit du master FOAD, soit de la DIFOR, est affecté à chaque groupe.

La formation s'étale ensuite sur 12 semaines. Les 10 premières semaines sont consacrées au travail collectif. Chaque groupe élabore son projet, depuis la définition $\mathrm{du}$ sujet jusqu'à sa réalisation technique qui est publiée aux vues de tous les 
participants Inti en semaine 9. À titre d'illustration, les sujets choisis en 2005-2006 portaient sur les mathématiques collaboratives à distance, un jumelage électronique entre un collège français et un collège marocain, un espace d'aide aux élèves atteints de phobie scolaire. En fin de semaine 10, chacun (apprenants et tuteurs) vote pour l'un des travaux de groupe suivant des critères de qualité qui correspondent au cahier des charges explicité dans le guide pédagogique. Le travail collectif dans les groupes s'accomplit dans les deux plates-formes Dokeos et Centra.

\subsection{Les types de supports pour le réflexif et le cadre dialogique}

Le processus réflexif, quant à lui, utilise trois types de supports : tuteur, de sa contribution personnelle; description de l'évolution de ses représentations entre le début et la fin du travail collectif. Chaque tuteur rédige également un rapport réflexif sur le fonctionnement de son groupe et analyse son rôle. Les rapports sont remis aux deux coordinateurs institutionnels (DIFOR et master) qui ne sont pas en position de tuteurs. L'anonymat des contributions est ainsi préservé.

- Une synthèse des rapports publiée par les coordinateurs en début de semaine 11. Elle est constituée de larges extraits des rapports réflexifs, classés par groupe. À cela s'ajoutent également des extraits d'un autre type de document, que nous n'avons

- Un blogue individuel dont l'accès en lecture et écriture de commentaires est paramétrable. Chacun décide s'il veut autoriser en tout ou en partie des participants Inti, voire n'importe quel internaute, à lire et $^{3}$ à commenter son blogue. Ce paramètre d'ouverture de l'accès est modifiable à tout moment. Chaque apprenant et chaque tuteur gère son blogue. S'il est demandé à chacun de l'ouvrir dès l'étape de préparation et d'y tenir, en particulier, un journal de bord, son auteur peut y écrire à sa guise et le continuer après la formation (voir la dernière ligne en continu et pointillés de la Figure 1).

- Un rapport réflexif individuel remis aux coordinateurs de la formation en semaine 10. Chaque apprenant rédige dans un format libre un document abordant les points suivants: analyse du fonctionnement du groupe, du rôle du

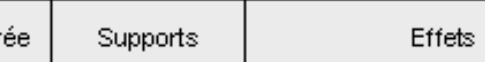

\begin{tabular}{|c|c|c|}
\hline Dates, durée & Supports & Effets \\
\hline s1 às9 & blogue & $\begin{array}{l}\text { Gestion émotionnel, stress, } \\
\text { action : rétrospectif, prospectif }\end{array}$ \\
\hline s9 à $\$ 12$ & $\begin{array}{l}\text { rapport réflexif, } \\
\text { blogue, forum }\end{array}$ & $\begin{array}{c}\text { De l'imaginé au vécu, bilan, } \\
\text { constater évolution } \\
\text { représentations, } \\
\text { préparation au transfert }\end{array}$ \\
\hline indéterminée & blogue & transfert \\
\hline
\end{tabular}

pas mentionné jusqu'à présent, le rapport d'analyse du travail collectif d'un autre groupe rédigé par chaque apprenant et remis au coordinateur. Cette synthèse joue donc un rôle important. Elle donne un retour sur le rapport réflexif, montre les disparités (ou convergences) de points de vue au sein d'un même groupe quant au vécu, ainsi que celles se rapportant à la perception de la qualité des travaux collectifs. Le tout en préservant l'anonymat des propos. À la suite de la publication de cette synthèse, chaque groupe est invité à faire le bilan de la formation de façon collective, soit dans le forum du groupe (ou dans le forum général Inti), soit lors d'une séance synchrone Centra. 
À travers ces différents supports et leurs fonctionnalités respectives, nous avons donc inscrit d'emblée le processus réflexif dans un cadre dialogique qu'illustre la Figure 2. Chaque auteur, apprenant ou tuteur, en contrôle partiellement l'ouverture sociale.

\subsection{Les différentes dimensions du travail réflexif}

La Figure 1 aligne sur l'axe du temps les différentes dimensions $\mathrm{du}$ travail réflexif qui peuvent apparaître dans le scénario que nous venons de décrire. Suivant Killion et Todnem (cité par Makinster et al., 2006), cela concerne :

- une réflexion dans la pratique (réflexion-dans, cf. reflection-in-practice). Dès l'instant où l'auteur du blogue publie ses premiers messages jusqu'en fin de travail collectif (semaine 9), ce support de pensée passant par l'écrit lui permet non seulement de faire le point sur ce qu'il est en train de faire et de vivre au sein de son groupe, mais aussi de se préparer aux étapes suivantes de son travail.

- $\quad$ une réflexion sur la pratique (réflexion-sur, cf. reflection-on-practice) entre la fin du travail collectif et celle de la formation (semaines 9 à 12). C'est le moment où l'on passe de l'imaginé au bilan du vécu et où l'on constate l'évolution de ses représentations. Ceci s'exprime dans le rapport réflexif, ou encore, après la publication de la synthèse, dans le blogue ou les forums.

- une réflexion pour la pratique (réflexion-pour,

cf. reflection-for-practice) de l'ouverture du blogue jusqu'après la session de formation. Parallèlement à la tenue d'un fil de messages dans le blogue concernant Inti, chaque

\begin{tabular}{|c|c|c|c|c|c|c|}
\cline { 4 - 7 } & & & \multicolumn{3}{|c|}{ Blogues } & \\
\hline Groupes & Membres & $\begin{array}{c}\text { Forums } \\
\text { (messages) }\end{array}$ & Messages & Commentaires & Réflexion-pour & $\begin{array}{c}\text { Rapport } \\
\text { réflexif }\end{array}$ \\
\hline G1 & 5 & 342 & 53 & 12 & 2 & 5 \\
\hline$G 2$ & 4 & 126 & 67 & 19 & 2 & 4 \\
\hline$G 3$ & 5 & 246 & 60 & 25 & 2 & 5 \\
\hline$G 4$ & 5 & 181 & 37 & 6 & 2 & 5 \\
\hline
\end{tabular}

Tableau 2. Vue d'ensemble de la participation au processus réflexif pour tous les acteurs auteur a la possibilité d'ouvrir d'autres fils de discussion sur des sujets libres, personnels ou professionnels. La gestion de ces fils peut avoir lieu avant le début de la formation, pendant et après, puisque le blogue comme le courriel, n'étant pas des outils institutionnels, restent ouverts en dehors de ces cadres institutionnels.

\subsection{L'organisation spécifique des tuteurs}

Comme nous l'avons vu, apprenants et tuteurs sont soumis aux mêmes exigences de conduite de processus réflexif (à quelques variantes de contenu près). Cela s'explique, d'une part, par le fait qu'une partie de ces tuteurs sont novices. Le processus réflexif soutient alors l'apprentissage. D'autre part, ces exigences communes estompent la frontière entre les statuts de ceux qui sont en position d'apprenant et de ceux à qui est dévolu un rôle de tuteur. On se rapproche d'un processus de compagnonnage (Reffay et Chanier, 2005) entre apprenant, tuteur novice et tuteur confirmé.

Cependant, les tuteurs ont bien un rôle distinct des apprenants, celui de soutien aux individus du groupe, d'animation et d'organisation du travail $\mathrm{du}$ groupe. Une organisation spéciale leur est donc offerte. Ils participent au groupe de conduite de la formation, qui mélange tuteurs et coordinateurs, et échangent en continu au moyen d'un forum privé de la plate-forme Dokeos et d'une série de réunions synchrones Centra avant, pendant et après la session de formation. Ils participent ainsi à la constitution des groupes, à la présélection des thèmes et à la coordination entre les groupes. 
Outre les fonctions de compagnonnage des tuteurs novices et de coordination de la formation, ce groupe a une autre fonction essentielle, à savoir gérer l'interculturalité institutionnelle. Nous avons déjà mentionné l'intérêt d'introduire un mélange des cultures d'apprentissage et institutionnelles pour le développement professionnel des enseignants. Cependant, les travaux de recherche montrent que les échecs de télécollaboration mêlant individus d'institutions différentes sont nombreux. Les difficultés proviennent en premier lieu des conflits entre les cultures différentes des enseignants (tuteurs) impliqués, le développement de la compétence interculturelle des formateurs faisant encore l'objet de trop peu d'attention dans le milieu éducatif (Audras et Chanier, sous presse).

\subsection{De la conception au vécu}

Après avoir décrit les différents composants et fonctions du processus réflexif, tel que conçu par nous, concepteurs de la formation, voyons comment les participants, qu'ils soient apprenants ou tuteurs, s'en sont emparés.

\section{Le vécu du processus réflexif lors de la session de formation}

\subsection{Données quantitatives}

Vingt-et-un apprenants étaient initialement inscrits à la session de formation Inti en 2005-2006. Six enseignants de la DIFOR ont abandonné lors des premières étapes : 2 durant la phase de préparation, par suite de changements survenus dans leur charge professionnelle habituelle; 4 durant les deux premières semaines, phase déterminante où les groupes se transforment en communauté d'apprentissage. Il semble que certains aient été intéressés à venir «voir» ce que pouvaient être de nouveaux environnements technologiques, sans vouloir pour autant entrer dans une démarche d'apprentissage, ce qui pourtant était explicité dans le contrat d'inscription.
Ce sont donc finalement 15 apprenants, 7 enseignants DIFOR et 8 stagiaires du master qui ont suivi cette session. Ils étaient répartis dans 4 groupes animés par 3 tuteurs DIFOR et un quatrième enseignant de l'université.

Le Tableau 2 apporte une vue d'ensemble, par groupe, de la participation de tous les acteurs au processus réflexif. Tous (apprenants et tuteurs) ont rendu leur rapport réflexif. Un nombre significatif de messages ont été postés dans les blogues, qui ont suscité des commentaires (moins dans le 4 e groupe). Dans chaque groupe, 2 personnes ont donné à leur blogue une dimension réflexif-pour. Nous avons également fait figurer une colonne récapitulant le nombre de messages postés par les membres de chaque groupe dans leurs forums de groupe. Ces forums ne font évidemment pas partie du processus réflexif, mais sont au contraire un lieu d'échanges et de travail entre membres d'un même projet collectif. Le rapport entre ces 3 colonnes (messages de forum, messages de blogues et commentaires de blogues) indique que les participants ont bien su faire la part des choses entre ces deux modalités textuelles (forum et blogue), tous deux supports de discussion, en ne mélangeant pas le réflexif avec le collaboratif. La participation dans l'un ne s'est pas faite au détriment de la participation dans l'autre.

Le Tableau 3 donne le détail par individu. Rappelons que nous avions, en tant que concepteurs de la formation, laissé une grande liberté dans les formes d'usage des blogues. Précisons enfin que personne n'avait d'expérience d'écriture dans un blogue.

Une seule personne n'en a pas ouvert (ou l'a rendu inaccessible), en préférant tenir en privé son journal de bord, la réflexion-dans lui paraissant a priori trop difficile.

(1) La définition et l'utilisation du blogue devraient être davantage précisées. C'est un espace que j'ai peu utilisé car il imposait un 
double recul. J'ai par conséquent utilisé un carnet de bord en parallèle du blogue pour consigner un premier niveau de réflexion illustré par des impressions notées dans le feu de l'action. Ces réflexions donnent lieu à un travail d'analyse approfondie plutôt en fin d'expérience (Fiche Réflexive). Dans un espace ouvert de publication tel que le blogue, ces impressions ne peuvent être consignées de façon brute et supposent un premier travail d'analyse $\mathrm{du}$ processus collaboratif plus exigeant et difficile à mener en parallèle de «l'action » basée sur la construction d'un produit.

[extrait de rapport réflexif, apprenant, 10 mars]

\begin{tabular}{|c|c|c|c|}
\hline Individus & Messages & Commentaires & Réflexion-por \\
\hline G1_AP1 & 14 & 2 & 1 \\
\hline G1_AP2 & 33 & 3 & 1 \\
\hline G1_AP3 & 6 & 4 & 0 \\
\hline G1_AP4 & inaccessible & & \\
\hline G1_TUT & 5 & 3 & 0 \\
\hline G2_AP1 & 3 & 2 & 0 \\
\hline G2_AP2 & 23 & 11 & 1 \\
\hline G2_AP3 & 16 & 5 & 0 \\
\hline G2_TUT & 25 & 1 & 1 \\
\hline G3_AP1 & $\begin{array}{l}\text { non ouvert/ } \\
\text { inaccessible }\end{array}$ & & \\
\hline G3_AP2 & 33 & 5 & 0 \\
\hline G3_AP3 & 16 & 10 & 0 \\
\hline G3_AP4 & 0 & 0 & 1 \\
\hline G3_TUT & 11 & 10 & 1 \\
\hline G4_AP1 & 2 & 1 & 0 \\
\hline G4_AP2 & 14 & 3 & 1 \\
\hline G4_AP3 & 16 & 0 & 1 \\
\hline G4_AP4 & inaccessible & & \\
\hline G4_TUT & 5 & 2 & 0 \\
\hline
\end{tabular}

Tableau 3. Détails quantitatifs par blogue

Fait remarquable, l'ensemble des participants (moins 3 personnes) ont ouvert l'accès à leur blogue à tout l'Internet. Ce n'est pas nécessairement un choix qui s'est opéré d'emblée, comme nous le verrons. La variance de participation entre individus est importante, mais pratiquement tous ont reçu des commentaires (sauf deux, dont un qui ne l'a pas du tout utilisé et s'est marginalisé par ailleurs dans son travail collaboratif). La fonction dialogique a bien fonctionné. Les commentaires ont été postés par des pairs du groupe, par le tuteur du groupe ou par des membres d'autres groupes ${ }^{4}$.

\subsection{La fonction auteur et le scénario d'écriture dans un blogue}

Le parcours des différents blogues livre des histoires assez similaires que l'on peut tenter de synthétiser sous forme de scénario. Il laisse voir les démarches d'entrée en processus réflexif.

À l'ouverture du blogue, les tout premiers messages sont d'abord des essais en privé. On essaie les fonctionnalités techniques de l'outil. Certains s'interrogent sur les fonctions communicationnelles qu'ils vont utiliser. Transparaissent alors les positionnements a priori et subjectif des individus.

(2) Prise de tête

Je crois que je ne suis pas douée pour la création de blogue...

[blogue tuteur 1 , décembre]

(3) Pratique le blogue pour stocker ses impressions! Finalement, j'aurai peut-être du mal à m'en passer ...

[blogue tuteur 2, décembre]

(4) En attendant que "la mayonnaise prenne", j'ai confiance dans nos réciproques et bénéfiques influences.

[blogue apprenant, décembre]

Immédiatement, l'utilisateur prend conscience de ce que Soubrié (2006) qualifie de retour en force grâce au blogue de la "fonction auteur». On s'exprime d'abord pour soi, en privilégiant la fonction communicative du journal ou carnet de bord, ainsi que la dénomment explicitement les auteurs. Les messages prennent la forme de comptes rendus très factuels (cependant importants pour l'autoapprentissage). Rapidement, chacun ressent le besoin d'exprimer des réactions et sentiments très personnels. Durant cette première période, une 
fraction notable des blogues n'est pas ouverte en accès libre sur la Toile.

Avec l'expression des impressions personnelles, se pose la question de la «figuration de soi » (Soubrié, 2006). Le journal intime doit-il devenir «extime», suivant la terminologie usitée pour qualifier les blogues sur la Toile (Soubrié, 2006)? La question émerge dans les forums des différents groupes: doit-on / a-t-on intérêt à ouvrir l'accès à son blogue aux membres de son groupe? À tous les participants Inti? À tout l'Internet? On observe également une curiosité quant au processus réflexif des autres. Aucune consigne officielle n'est donnée en réponse. Certains donnent l'exemple en ouvrant largement leur blogue. L'immense majorité suit alors. On écrit sur soi, mais on sent que l'on a intérêt à écrire pour être lu, dans une perspective de dialogue, tout hypothétique encore à ce moment-là. C'est l'acte fondateur de la rentrée en processus réflexif, le premier changement de position, qui s'accomplit dans un contexte social, et qui va de pair avec une perception différente de l'apprentissage collaboratif dans les autres espaces dédiés.

(5) Je définirai mes premières interventions dans les espaces collectifs d'échanges synchrones et asynchrones comme «frileuses ». [...]. Je mettrai ce constat en perspective avec les premiers messages postés dans le blogue qui marquent en réalité les hésitations à «ouvrir» et faire découvrir un espace de réflexion personnel. J'ai d'ailleurs tenu un carnet de bord en parallèle du blogue. L'attitude et l'enthousiasme d'un collaborateur m'ont permis de dépasser cette représentation initiale qui a finalement abouti à une coproduction plutôt réussie. [rapport réflexif, apprenant, 10 mars]

L'ouverture permet à chacun d'aller lire les autres, d'emprunter. Les modèles de journaux de bord s'échangent. La variété des formes d'interventions dans les blogues encourage les prises de risque. Un apprenant qui n'avait pas encore ouvert son blogue passe à l'acte.

Les premiers commentaires font leur apparition dans quasiment tous les blogues. On écrit maintenant en sachant que l'on sera sans doute lu, sans modifier pour autant la façon très personnelle de s'exprimer. Les commentaires des autres soutiennent, partagent, contredisent.

(6) L'entraide était de rigueur et souvent le blogue était un bon indicateur de l'état d'esprit de chacun à l'issue d'une activité, d'une étape qui venait de s'achever. [rapport réflexif, apprenant, 10 mars]

(7) Ce qui me surprend davantage, c'est le vote sanction que l'on a fait subir au groupe $3 .$. pas une voix pour la question relative à l'interface. Après, on me dira que le vote s'appuie sur des critères objectifs définis par le guide pédagogique Inti. [...] Pour ma part, j'ai été très subjectif dans mon vote. Mais alors me direzvous? Pourquoi reprocher aux autres ce que je fais moi-même? [blogue, apprenant 1, 6 mars]

(8) Je crois que tu es trop sévère avec toi-même lorsque tu t'accuses de subjectivité. J'ai déjà en partie répondu sur ce sujet dans mon blogue, mais je ne résiste pas à l'envie d'en remettre une couche. J'ai moi aussi voté pour notre groupe et sans faire de détails. [...] Subjectif, moi? oui, et alors? c'est juste le signe que j'ai apprécié ce qu'on a construit (y compris les relations entre participants) et surtout ce que le groupe m'a apporté. [commentaire tuteur, 6 mars]

Les exemples 7 et 8 témoignent d'une réaction après la publication des votes et de la synthèse. Même si la discussion a commencé dans le forum du groupe, l'apprenant 1 laisse ici transparaître plus directement son ressentiment, en sachant que cela n'a pas la même répercussion que s'il était formulé 
en l'état dans le forum. La mise en mots est une étape importante pour entamer la réflexion (et porter ici un point de vue qui se distingue du prescrit), qui trouve immédiatement un écho de la part de l'autre (en l'occurrence le tuteur). Celui-ci appuie cette démarche d'extériorisation en livrant également un point de vue très personnel. La procédure de vote, point d'aboutissement du travail d'inspection des travaux respectifs, a suscité beaucoup d'interrogations (en positif ou négatif). Ces messages et les commentaires associés sont une façon de se rassurer avec l'autre, en dehors des espaces du groupe.

\subsection{Les dimensions du travail réflexif}

Reprenons les dimensions du travail réflexif en commençant par la réflexion-dans. Nous venons de donner des exemples d'expressions d'émotions que l'on peut considérer comme exerçant un rôle de régulation sur le ressenti de l'individu en réponse immédiate à des évènements de la formation. Outre la fonction de réassurance déjà mentionnée, les messages dénotent souvent une fonction de préparation à la suite. Dans (11), l'apprenant à la fois se prépare à gérer ses émotions lors d'une prochaine réunion de groupe et planifie ses actions.

(11) J'ai l'impression que le dynamisme du groupe s'étiole sensiblement alors que nous démarrons l'étape $3 \mathrm{du}$ projet. Espérons que nous réussirons à remplir le contrat... On se rendra plus compte des avancées de chacun ce soir, durant la séance que j'organise. Pour ma part, [...] je fais de mon mieux pour participer le plus possible aux forums... et j'ai un doute quant à l'efficacité de mes interventions. Il faudrait sans doute prévoir d'échanger des documents par email pour travailler plus efficacement, en discuter ce soir .... [blogue apprenant 4, 31 janvier]

Après avoir animé cette séance synchrone sur Centra (tâche difficile, accomplie à un moment crucial de la vie du projet collectif), l'apprenant revient en parler.

(12) Ca y est, c'est fait! J'éprouve enfin un grand soulagement. J'avoue que les compétences de chaque participant m'impressionnent à chaque fois. [...] Parfait! Je sens comme XXX que le groupe s'est consolidé durant cette $4^{\mathrm{e}}$ séance, ce qui me rend plus optimiste. À suivre... [blogue apprenant 4, 2 février]

En ce qui concerne la réflexion-sur, les rapports réflexifs témoignent de l'intense activité de réflexion à laquelle se sont livrés tous les participants (apprenants DIFOR et master, tuteurs) sur tous les points prescrits. En tant que chercheur, on peut se demander si la qualité de ces rapports est en partie motivée par le fait qu'ils ne seront pas écrits «dans le vide », mais en sachant par avance que de larges parts seront exposées à tous. Pour y répondre, il faudrait notamment savoir jusqu'à quel point et comment la synthèse sur ces rapports a été lue. Les exemples représentant ce travail réflexif ne manquent donc pas dans ces rapports. Nous n'en citerons que deux qui serviront de transition vers la dernière dimension, car déjà leurs auteurs en tirant leurs bilans se projettent vers l'avenir.

(13) Cette expérience a fait évoluer positivement mes représentations initiales d'une telle formation. Mes craintes se sont peu à peu dissipées: le travail à distance est possible avec des personnes d'univers fort différents et le travail coopératif peut rapidement se transformer par la volonté commune en un travail collaboratif de qualité. [...]. Nos apprentissages en matière de négociation et de collaboration sortent nettement grandis de cette expérience. [rapport réflexif, apprenant DIFOR, 10 mars]

(14) Je savais par expérience (Inti 2005 comme apprenant) que cette opération était une remise 
en question des méthodes habituelles de travail pour un enseignant: pas de présentiel, des outils nouveaux, une relation différente avec les participants. Cependant [...], je ne mesurais pas à quel point je serais amené à me poser des questions. Des questions, en premier lieu, sur le rôle que doit jouer le tuteur d'un groupe : [...]. En fait, ma première interrogation portait sur ma compétence à remplir toutes ces tâches. Et puis, j'ai eu l'impression que c'était le groupe qui faisait le tuteur [...] Alors, c'est vrai que j'ai fini par considérer notre groupe comme une entité nouvelle, une sorte de famille à laquelle on appartient avec plaisir. [rapport réflexif, tuteur DIFOR, 10 mars]

La dernière dimension $\mathrm{du}$ travail réflexif, la réflexion-pour, apparaît nettement dans ces rapports. Les participants, en faisant leur bilan, font ressortir des points qu'ils jugent essentiels et expriment le fait qu'ils voudraient poursuivre ce type d'expérience. C'est autre chose de le formuler en des termes plus finalisés de transfert entre cette formation et leur situation d'enseignant habituelle. Il faut pour cela du temps et, sans doute aussi, des occasions. La situation est évidemment bien différente pour les stagiaires du master pour lesquels la distance est inscrite dans leur avenir professionnel. Cependant, on peut voir apparaître dans les blogues les prémisses de cette réflexionpour. Dans les tableaux 1 et 2, nous avions listé les individus (DIFOR et master) qui avaient utilisé ce support pour d'autres sujets qu'Inti. Les cas recensés correspondent aux situations suivantes: ouverture pendant ou après la formation de nouvelles catégories de messages sur des sujets se rapportant à d'autres formations dans lesquelles ils sont engagés, de sujets portant sur les différents usages des TICE, en s'appuyant sur les découvertes de l'environnement Inti afin de se projeter vers ceux qui pourraient s'appliquer dans leurs établissements scolaires ou en formation continue avec d'autres enseignants.

\subsection{Réflexif, communauté d'apprentissage et de pratique}

Dans les thèmes du réflexif que nous avons illustrés jusqu'à présent, se retrouvent bien les composantes de base de la dynamique de collaboration (Henri et Lundgren-Cayrol, 2001, p.100), coordination, engagement, communication (dans ce dernier composant, il est question de contenus et liens entre ces idées à propos du projet collectif, thème peu cité ici, bien présent tout en étant minoritaire, dans les messages). Mais, alors que dans les espaces collectifs on agit sur le projet, ici on en parle avec ses mots. Ce travail permet à l'individu de mieux prendre conscience de son apprentissage individuel. Sans cela, c'est le volet collectif que l'individu perçoit en premier. Le réflexif, en mettant le doigt sur ces interrelations, accélère la transformation du groupe en communauté d'apprentissage. Le phénomène est encore plus net quand le dialogue s'installe au sein du réflexif. C'est l'autre alors qui renforce la perception des liaisons entre l'individu et le groupe.

Qu'en est-il de la CdP? Le lecteur aura peut-être remarqué que, dans la figure 1, la forme géométrique symbolisant la $\mathrm{CdP}$ recouvre largement celle de la communauté d'apprentissage. En effet, les critères généralement retenus par la littérature pour distinguer les participants des communautés d'apprentissage de ceux des $\mathrm{CdP}^{5}$ ont ici des contours beaucoup plus ténus que dans des projets collaboratifs classiques. L'exemple suivant le fait sentir. Il s'agit du commentaire posté par le tuteur, après la séance tant redoutée par l'apprenante (voir 11 et 12 ), séance qu'elle devait animer.

(15) Bonsoir (apprenant 4), et bienvenue au club des accros à Centra. C'est un moment inoubliable, cette première fois où l'on emmène une réunion synchrone. Et tu ne contredis pas la règle qui veut que le (la) présentateur (trice) soit le seul à avoir un doute quant à sa 
Figure 3. Relation entre processus réflexif, communauté d'apprentissage et communautés de pratique (extension du modèle de Keiny (1996), « E » représente l'enseignant)
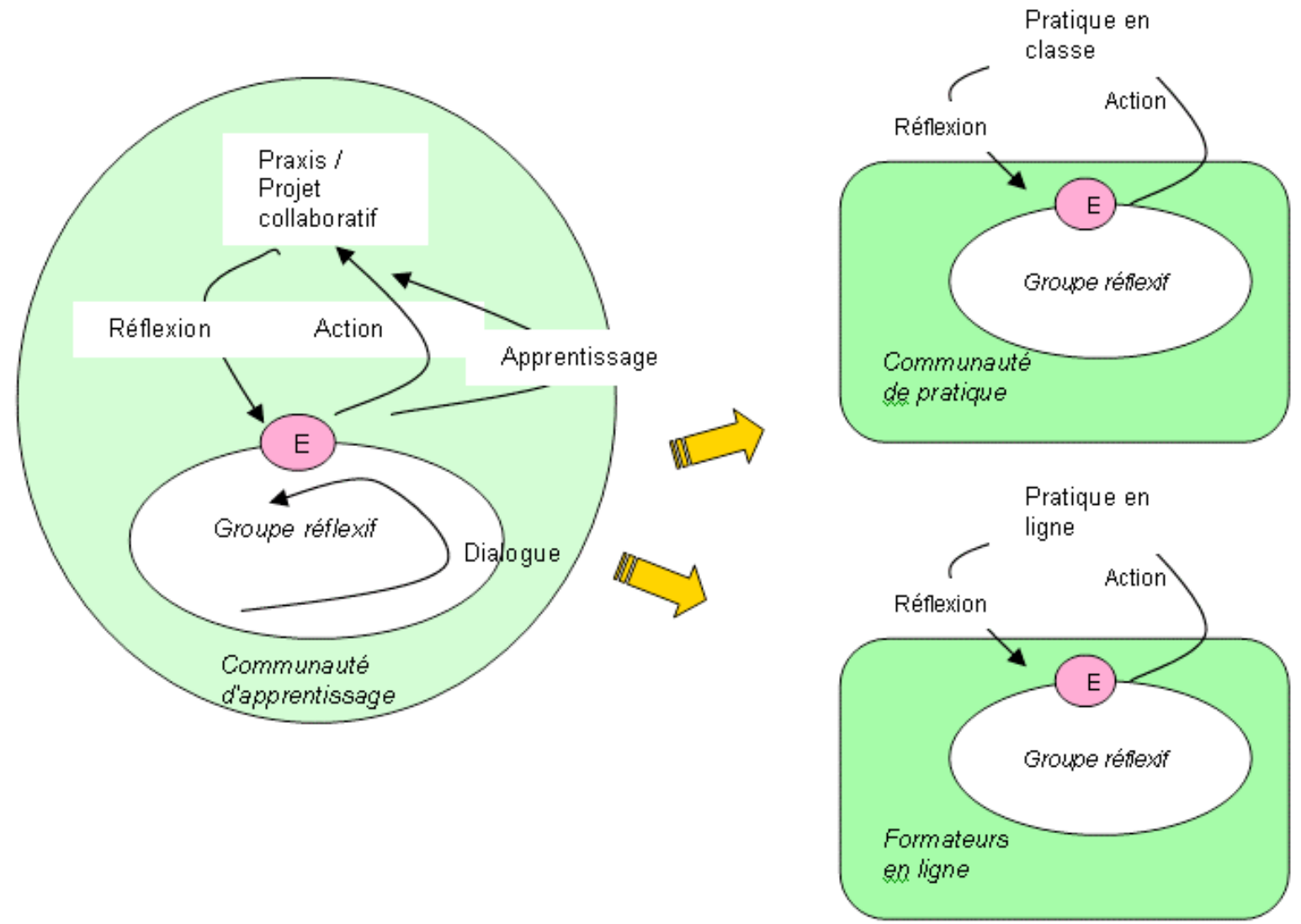

prestation : je trouve que tu t'en es tirée avec les honneurs (j'en ai même parlé dans mon blogue...). Moi aussi, je pense que le groupe a réussi à repartir et que le projet aboutira. [commentaire sur 12, tuteur, 3 février]

Ainsi donc, le praticien plus expérimenté reconnaît l'entrée du novice dans la communauté des formateurs en ligne. Nous ne sommes plus dans un simple rapport apprenant-tuteur, mais formateurformateur, et ce, dans un contexte qui dépasse la durée de cette formation.

L'expérience, intensive, vécue dans cette formation sert donc d'introducteur dans cette CdP en ligne. Mais quelle est cette communauté? Est-elle unique ou plurielle? Au contraire des exemples cités aux États-Unis, ses frontières ne se définissent pas à travers une géographie locale ou disciplinaire. Si l'on considère l'institution Éducation Nationale en France limitée au secteur de l'enseignement secondaire, la sous-direction des TICE au ministère a suscité, à travers la mise en place d'une plateforme technologique unique sur le plan national (qui rassemble un ensemble de plates-formes synchrones et asynchrones, dont Centra) et le soutien à un ensemble de projets de formation continue des enseignants par l'intermédiaire des réseaux (projets académiques, nationaux, européens, etc.), l'émergence d'une $\mathrm{CdP}$ de formateurs de formateurs en ligne. La formation Inti, au cours de ces deux années d'existence, a clairement recruté, pour ce qui est de la DIFOR, dans un milieu d'enseignants attirés, plus ou moins consciemment, par cette communauté. Elle a 
contribué à les former sur les deux niveaux de compétences évoqués (apprenant-formateur en ligne et tuteur en ligne). Sitôt les sessions Inti terminées, une partie de ces enseignants ont manifesté le désir ou ont effectivement participé à l'animation d'autres formations continues en ligne, tout en continuant à exercer leur métier d'enseignant en classe dans leur discipline respective.

Cette communauté a-t-elle des frontières institutionnelles (est-elle limitée aux membres de l'éducation nationale)? En règle générale la réponse sera affirmative, car le poids des institutions, des corps professionnels attachés, est déterminant dans la vie quotidienne. Au niveau de la petite échelle Inti, la question ne semble pas se poser en ces termes institutionnels. En témoigne cet indice : un an après cette formation, une stagiaire du master qui venait de réussir à monter son entreprise privée de FAD en Afrique du Nord a choisi de l'annoncer d'abord à une sélection de sa promotion et aux enseignants DIFOR de son groupe Inti. Cette stagiaire avait quitté la France peu après la session Inti, au contraire des enseignants qui sont restés dans leurs établissements respectifs.

La Figure 3 résume notre façon de percevoir les relations entre processus réflexif, communauté d'apprentissage et communauté de pratique au sein des communautés de formateurs.

\section{Conclusion}

Nous avons défendu l'idée que la conduite d'une pratique réflexive sur les pratiques et la participation à une $\mathrm{CdP}$ en ligne sont deux activités qui peuvent être menées conjointement par l'enseignant dans une perspective de développement professionnel. De récentes politiques éducatives orientent la formation initiale et continue des enseignants vers le travail collectif en réseaux. S'offre alors la possibilité de concevoir des formations qui ont pour enjeux: d'être un terreau favorisant l'émergence de $\mathrm{CdP}$ en ligne; d'ouvrir les praticiens à de nouvelles postures pédagogiques impliquant une certaine rupture avec l'habitus sur le plan des formes de travail (travail avec des collègues et non seul, avec de nouveaux outils collecticiels); de les familiariser avec des formes de pédagogie peu pratiquées dans le système éducatif actuel (approches socioconstructivistes de l'apprentissage).

Pour ce faire, nous avons émis l'hypothèse que plonger des enseignants dans la position d'apprenants, avec l'objectif de se constituer en communauté d'apprentissage autour d'un projet collaboratif en ligne, représenterait une expérience, mobiliserait des connaissances et un ensemble de valeurs susceptibles de leur ouvrir la porte d'entrée étroite à des $\mathrm{CdP}$ en ligne. Pour soutenir ce travail exigeant sur le plan de l'engagement, nous avons montré l'intérêt de lui adjoindre un processus réflexif avec un double objectif; d'une part, mettre l'enseignant (apprenant ou tuteur novice) en position de saisir les liens profonds existant entre l'apprentissage individuel et celui du groupe et d'accélérer ainsi la délicate organisation du groupe en communauté d'apprentissage; d'autre part, de former cet enseignant à la pratique réflexive en lui fournissant des outils, des méthodes et un cadre valorisant cette démarche, afin qu'elle perdure dans celui de sa future CdP. Un rappel des premiers résultats de recherche indique que cela présuppose de concevoir cette mise en réflexion sur les pratiques à travers un cadre dialogique avec les pairs.

Nous avons ensuite présenté un dispositif original de travail réflexif intégré au sein d'une formation collaborative avec ses supports d'expression, dont le blogue, son scénario et son processus dialogique. Ce dispositif est destiné à soutenir trois modalités de travail réflexif, que nous avons développées. L'étude de cas d'une session de formation mélangeant enseignants du secondaire et formateurs en stage à l'université a montré à quel 
point les participants (tuteurs et apprenants), tous novices, se saisissaient de ces outils et développaient un processus de qualité, en relation étroite avec leur engagement dans les projets collectifs. Des exemples nombreux ont en particulier illustré le fait que le blogue, doté d'une telle scénarisation, était un outil de première importance à travers lequel s'organisait le dialogue en relation avec le travail réflexif. Nous avons enfin pointé certains traits de cette communauté d'apprentissage qui la rapprochaient singulièrement d'une CdP. Cet exemple apporte un élément de réponse à la difficile constitution d'une CdP ex nihilo, constatée dans la littérature de recherche.

On appréciera aisément les limites de cet article. Il cherche à introduire dans un même paradigme orienté vers le développement professionnel de l'enseignant les notions d'apprentissage collaboratif, de communauté de pratique, de réflexion sur les pratiques et de cadre dialogique autour du réflexif. Il présente ensuite un dispositif de processus réflexif au sein d'une formation. Une étude de cas ne peut prétendre qu'à être une première illustration de faisabilité de mise en correspondance de l'ensemble. Le paradigme général mérite d'être plus amplement discuté, le dispositif réflexif, d'être répliqué.

À cette occasion, on pourra en particulier se demander s'il vaut mieux, comme ici, laisser apprenants et tuteurs découvrir par eux-mêmes les usages qu'ils peuvent faire du blogue et de ses conditions d'accès, ou scénariser davantage cette partie en indiquant des préférences. Cela revient à se demander si, un cadre général étant fixé (supports multiples, cadre dialogique), la mise en démarche réflexive de l'individu requiert un espace de choix, décisions, découvertes (donc une réflexion sur la réflexion) ou si elle gagne à être plus encadrée.

D'autre part, des études longitudinales sur quelques individus en cours de formation et post-formation mériteraient d'être conduites en vue de savoir si la pratique réflexive perdure avec ou sans intégration dans une CdP. Nous n'avons pu avancer ici que des bribes d'indices. De même, la question de la valeur que les participants à la formation accordent à ce processus réflexif n'a pas fait l'objet d'un repérage spécifique (au moyen, par exemple, de questionnaires post-entretiens). Sur ce point, nous terminerons en apportant un indice en ce sens témoignant d'une réflexion (non sollicitée) de méta niveau sur le processus réflexif. En (16), un apprenant DIFOR, qui quelques années auparavant avait été stagiaire du master et avait vécu un projet collaboratif un peu similaire (Learn-Nett), mais sans scénarisation spécifique du réflexif, s'exprime :

(16) Enfin, je considère le travail métacognitif comme une étape fondamentale de la formation, puisque c'est l'occasion de prendre $\mathrm{du}$ recul par rapport à soi et au groupe, de se questionner, et surtout de mesurer son évolution dans le temps (de l'appréhension à l'autonomie, en passant par l'affirmation de soi). Grâce à ce travail, je m'aperçois que ma capacité à travailler en groupe, et de surcroît à distance, s'est améliorée (comparé notamment à ma première expérience Learn-Nett). Je retiens de cette démarche que "collaborer", $c^{\prime}$ est contribuer aux apprentissages du groupe, et se nourrir, s'enrichir de la confrontation et de l'échange avec le groupe. [rapport réflexif, apprenant, 10 mars]

\section{Références}

Audras, I. et Chanier, T. (sous presse). Tridem, interactions à plusieurs à l'écrit - à l'oral et acquisition d'une compétence interculturelle dans une formation en langue en ligne. Lidil, 38.

Barab, S. A., Kling, R. et Gray, J. H. (dir.). 2004. Designing for virtual communities in the service of learning. Cambridge : Cambridge University Press.

Barab, S. A., MaKinster, J. G. et Scheckler, R. (2004). Designing system dualities: Characterizing an online 
professional development community. Dans S. A. Barab, R. Kling et J. H. Gray (dir.), Designing for virtual communities in the service of learning (p.53-90). Cambridge : Cambridge University Press.

Cartier, J. et Chanier, T. (2006). Le processus réflexif dans la formation de formateurs de type collaboratif en ligne (Rapport du Laseldi). Besançon, France : Université de Franche-Comté, Laboratoire de Sémiolinguistique, Didactique et Informatique. Récupéré le 8 mai 2007 du site Archive EduTice: http:/ / edutice.archives-ouvertes.fr/

Daele, A. et Charlier, B. (dir.). (2002). Étude: Les communautés délocalisées d'enseignants (Programme Numérisation pour l'Enseignement et la Recherche : Observation des usages et des pratiques dans le domaine de l'enseignement scolaire, volet Usages et normes). Paris : Maison des Sciences de l'Homme de Paris. Récupéré le 8 mai 2007 du site Archive EduTice : http://edutice.archives-ouvertes.fr/ $\underline{\text { edutice- } 00000388}$

Daele, A. et Charlier, B. (2006). Pourquoi les communautés d'enseignants aujourd'hui? Dans A. Daele et B. Charlier (dir.), Comprendre les communautés virtuelles d'enseignants. Pratiques et recherches (p. 83-104). Paris : L'Harmattan.

Ferraro, J. M. (2000). Reflective practice and professional development. ERIC Digest. Washington, DC: Education Resources Information Center (ERIC) Clearinghouse on Teaching and Teacher Education. (Numéro de service de reproduction de documents ERIC ED449120)

Henri, F. et Lundgren-Cayrol, K. (2001). Apprentissage collaboratif à distance. Pour comprendre et concevoir les environnements d'apprentissage virtuels. Sainte-Foy, Canada : Presses de l’Université du Québec.

Henri, F. et Pudelko, B. (2002). La recherche sur la communauté asynchrone: de l'outil aux communautés. Dans A. Daele et B. Charlier (dir.), Étude: Les communautés délocalisées d'enseignants (Programme Numérisation pour l'Enseignement et la Recherche : Observation des usages et des pratiques dans le domaine de l'enseignement scolaire, volet Usages et normes) (p. 12-44). Paris : Maison des Sciences de l'Homme de Paris. Récupéré le 8 mai 2007 du site Archive EduTice : http:/ / edutice.archives-ouvertes.fr/edutice$\underline{00000388}$

Keiny, S. (1996). A community of learners: promoting teachers to become learners. Teachers and teaching: Theory and Practice, 2(2), 243-272.

Lave, J. et Wenger, E. (1991). Situated Learning: Legitimate Peripheral Participation. New York : Cambridge University Press.

MaKinster, J. G., Barab, S. A., Harwood, W. S. et Andersen, H. O. (2006). The effect of social context on the reflexive practice of pre-service science teachers: Incorporating a Web-supported community of teachers. Journal of Technology and Teacher Education, 14(3), p. 543-579.

Ministère de l'éducation nationale, de l'enseignement supérieur et de la recherche - Sous-direction des technologies de l'information et de la communication pour l'éducation. (2005). Référentiel C2I niveau 2 enseignant. Paris: auteur. Récupéré le 8 mai 2007 du site du Certificat informatique et internet, section $\mathrm{C} 2 \mathrm{I}$ niveau 2 - enseignant : http:/ / c2i.education.fr/C2i2e/f_referentiel.htm

Perrenoud, P. (2001). Développer la pratique réflexive dans le métier d'enseignant. Professionnalisation et raison pédagogique. Paris : ESF.

Reffay, C. et Chanier, T. (2005). Approche minimaliste de la formation d'enseignants du supérieur au rôle de concepteur-tuteur de cours en ligne (Rapport du LIFC). Besançon, France: Université de Franche-Comté, Laboratoire d'Informatique de l'Université de Franche-Comté. Récupéré le 8 mai 2007 du site Archive EduTice: http://edutice.archivesouvertes.fr/ edutice-00000920

Wilhem, R.W., Coward, M.F. et Hume, L.M. (1996). The effects of a professional development institute on pre-service teachers' perceptions of their 
intercultural knowledge and diversity. Teacher

Educator, 32(1), p. 48-61.

Schlager, M. S. et Fusco, J. (2004). Teacher professional development, technology, and communities of practice: Are we putting the cart before the horse? Dans S. A. Barab, R. Kling et J. H. Gray (dir.), Designing for virtual communities in the service of learning (p.120-153). Cambridge: Cambridge University Press.
Soubrié, T. (2006, juillet). Le blogue : retour en force de la "fonction auteur». Communication présentée à la conférence JOCAIR : Journées Communication et Apprentissage Instrumentés en Réseau, Amiens, France.

\section{Notes}

${ }^{1}$ Dans cette troisième modalité, nous parlons d'éventualité et de potentialité, car, à la lecture de l'article, il semble que fort peu d'enseignants praticiens, non tuteurs des élèves, soient effectivement intervenus.

${ }^{2}$ Service de formation du personnel enseignant et administratif figurant dans chaque académie en France, l'académie définissant la zone géographique la plus importante de toute l'organisation de la vie éducative en France à tous les niveaux du primaire au supérieur.

${ }^{3}$ Ce paramètre de réglage des accès est la raison essentielle qui nous a fait choisir le système de blogue MSN® pour cette formation.

${ }^{4}$ Signalons toutefois deux internautes inconnus qui ont publié chacun un message très décalé dans deux blogues, ce qui a suscité des plaisanteries à l'intérieur de cette communauté.

${ }^{5}$ a) les premiers adhèrent librement tandis que les seconds sont plutôt contraints, un peu comme dans un «mariage arrangé ", b) le cadre des premiers est spécifique tandis que celui des seconds reste l'enseignement, et c) la durée du contrat pour les premiers n'est pas, comme c'est le cas pour les seconds, limitée a priori par l'institution. Cf. Reffay et Chanier (2005) pour une remise en contexte. 